\title{
The epidemiology, risk factors, and impact on hospital mortality of status epilepticus after subdural hematoma in the United States
}

\author{
Ali Seifi ${ }^{*}$, Ali Akbar Asadi-Pooya ${ }^{2,3}$, Kevin Carr $^{1}$, Mitchell Maltenfort ${ }^{4}$, Mehrdad Emami $^{2}$, Rodney Bell ${ }^{4}$, \\ Michael Moussouttas ${ }^{6}$, Moussa Yazbeck ${ }^{7}$ and Fred Rincon ${ }^{5}$
}

\begin{abstract}
Introduction: Subdural hematoma (SDH) is a well described risk factor in the development of Status Epilepticus (SE), however the epidemiology of SE after SDH is unknown. In this study, we sought to determine the epidemiology of SE, the prevalence of risk factors, and impact on hospital mortality using a large administrative dataset.

Methods: Data was derived from the Nationwide Inpatient Sample from 1988 through 2011. We queried the NIS database for patients older than 18 years, with a diagnosis of SDH and SE. Diagnoses were defined by ICD 9 CM codes $432.1,852.2,852.3$ and 345.3 for SE. Adjusted incidence rates of admission and prevalence proportions were calculated. Multivariate logistic models were then fitted to assess for the impact of status epilepticus on hospital mortality.
\end{abstract}

Results: Over the 23-year period, we identified more than 1,583,255 admissions with a diagnosis of SDH. The prevalence of SE in this cohort was $0.5 \%$ (7,421 admissions). The population adjusted incidence rate of admissions of SDH increased from $13 / 100,000$ in 1988 to 38/100,000 in 2011. The prevalence of SE in SDH, increased from $0.5 \%$ in 1988 to $0.7 \%$ in 2011. In hospital mortality of patients with SDH and without SE decreased from $17.9 \%$ to $10.3 \%$ while in hospital mortality of patients with SDH and SE did not statistically change. Mortality increased over the same period (2.3/100,000 in 1988 to 3.9/100.000 in 2011) and the diagnosis of SE increased mortality in this cohort (OR 2.17, $p<0.0001)$. The risk of SE remained stable throughout the study period, but was higher among older patients, blacks, and in those with respiratory, metabolic, hematological, and renal system dysfunction.

Conclusion: Our study demonstrates that the incidence of admissions of SDH is increasing in the United States. Despite a decline in the overall SDH related mortality, SE increased the risk of in-hospital death in patients with a primary diagnosis of $\mathrm{SDH}$.

Keywords: Subdural hematoma; Status epilepticus; Risk factors; Mortality

\section{Introduction}

Subdural hematomas (SDH) are most often described as resulting from traumatic brain injury (TBI) of varying degrees. In survivors of TBI, post traumatic epilepsy (PTE) is a well-known complication (Tomkins et al. 2008; Storti et al. 2012; Steg 1993; Hung and Chen 2012; Gupta and Gupta 2006; Agrawal et al. 2006), and

\footnotetext{
*Correspondence: seifi@uthscsa.edu

'Department of Neurosurgery, Division of Neurocritical Care, University of Texas Health Science Center at San Antonio, Mail Code 7843, 7703 Floyd Curl Drive, Medical School Building 102F, San Antonio, TX 78229-3900, USA Full list of author information is available at the end of the article
}

often complicates treatment in the hospitalized patient (Annegers et al. 1998). The epidemiology of status epilepticus in patients admitted with SDH is however poorly understood. Similarly, the pathophysiology of epileptogenesis in these patients is incompletely known, but is thought to be biphasic, with 1) early and 2) late post traumatic epilepsy (PTE) (Agrawal et al. 2006). One hypothesis states that in the immediate peri-injury period, brain swelling, cerebral ischemia and the release of excitatory amino acids and other toxins precipitate neuronal damage (Agrawal et al. 2006; Brodersen and Gjerris 1975). Comparatively, in the late post injury period, an excess of 
extracorpuscular hemoglobin facilitates the creation of cytotoxic hydroxyls, reactive oxygen species moieties and glutamate accumulation (Payan et al. 1970). These compounds collectively promote neuronal damage propagating the creation of an epileptogenic nidus, and occasionally leading to status epilepticus (Willmore et al. 1978; Rubin and Willmore 1980; Shaver et al. 1996; Sahuquillo-Barris et al. 1988; Becker 1986).

The diagnosis of status epilepticus in the setting of an extra-axial hemorrhage in general portends a poor prognosis (Dennis et al. 2002; Claassen et al. 2006; Little et al. 2007). In patients with acute or chronic SDH the risk of developing epileptiform activity is higher and increases in those requiring surgical evacuation (Yeh et al. 2013; Englander et al. 2003). In addition to high mortality and morbidity, the diagnosis of SDH presents a significant economic burden on healthcare systems and over the last several decades has been correlated to increases in health care costs (Frontera et al. 2011; Balser et al. 2013). Although, surgical intervention through trephination or craniotomy assisted evacuation has improved mortality in some cohorts, seizures and the diagnosis of status epilepticus (SE) may complicate recovery (Annegers et al. 1998; Rubin and Rappaport 1993; Cameron 1978; Drapkin 1991; Kotwica and Brzezinski 1991; Robinson 1984; Cole and Spatz 1961; Huang et al. 2011; Byung-Soo et al. 2008).

However, the epidemiology of status epilepticus (SE) after SDH has not been studied despite the fact that an association between SDH and seizures is widely demonstrated in academic literature (Rubin and Rappaport 1993; Cole and Spatz 1961; Rabinstein et al. 2010; Sabo et al. 1995; Ohno et al. 1993). Similarly, the risk factors for SE in patients with SDH have not been sufficiently characterized in previous literature (Huang et al. 2011; Westmoreland 2001; Wiedemayer et al. 2002). The purpose of this study was to determine the epidemiology of SE in patients with SDH and to characterize the associated risk factors for SE in these patients. We also investigated the impact of SE on in hospital mortality in patients with SDH.

\section{Materials and methods}

In this retrospective study, data was obtained from the Nationwide Inpatient Sample from 1988 through 2011. The NIS, a database maintained as part of the Healthcare Utilization Project of the Agency for Healthcare Quality and Research (HCUP-AHQR), is the largest allplayer inpatient database representing an approximate stratified $20 \%$ sample of all non-federal, short-term, general, and specialty hospitals serving adults in the United States.

The International Classification of Disease - Clinical Modification, 9th revision (ICD-9-CM) codes used to identify SDH patients [ICD-9-CM codes 432.1 (nontraumatic SDH) and codes 852.2 or 852.3 (traumatic $\mathrm{SDH})$ ] as previously reviewed in the literature (Frontera et al. 2011). These codes had a $70 \%$ sensitivity, $100 \%$ specificity, $94 \%$ positive predictive value, and 100\% negative predictive value when listed as the primary or secondary diagnoses (Frontera et al. 2011). This cohort included patients with acute, sub-acute and chronic SDH with the aforementioned admission ICD-9-CM codes Table 1.

We included patients 18 years of age or older, with a primary or secondary diagnosis of SDH. Patients' age, gender, and race (white, African-American, Asian-Pacific Islander, American/Indian Eskimo, other; and not stated) were obtained from the NIS database. From the cohort of patients with SDH, those who developed SE during their hospital admission, were identified using ICD-9CM code 345.3 (Claassen et al. 2007; Urtecho et al. 2013) as previously validated in the literature (Urtecho et al. 2013). A 2013 study by Urtecho and colleagues validated that the accuracy of the ICD-9-CM code for SE was $100 \%$ when defined as a neurologist's documentation of continuous clinical seizure activity for five minutes or longer, and/or two or more discrete seizures without interictal return to baseline (clinical diagnosis) and/or EEG consistent with SE per a board-certified neurophysiologist's interpretation (EEG diagnosis) (Urtecho et al. 2013). Based on clinical diagnosis alone, the ICD-9CM code for SE carried a sensitivity of $82 \%$ (95\% CI, $72-92 \%)$, specificity of $100 \%$, PPV $100 \%$, and NPV of $90 \%$ (95\% CI, 83-98\%); based on EEG data alone, the ICD-9CM code for SE carried a sensitivity of $55 \%$ (95\% CI, $42-67 \%)$, specificity of $100 \%$, PPV of $100 \%$, and NPV of 79\% (95\% CI, 69-89\%) (Urtecho et al. 2013).

In-hospital complications and organ failures that might have been related to the primary diagnosis, were identified among all patients, using the same ICD-9-CM codes as demonstrated in previous literature (Charlson et al. 1987; Quan et al. 2005; Rincon et al. 2012). Hospitals were divided by hospital location into: urban, sub-urban, and rural; by type into community (non-academic), academic (university-based), and public; and according to the Halpern criteria (Halpern et al. 2006), into small to medium size ( $<300$ beds), large (301-499 beds), and extralarge ( $>500$ beds).

National estimates were calculated according to accepted guidelines for the accuracy of NIS-HCUP data (Cameron 1978). That is, using the provided discharge weights, accounting for stratification, and clustering effects. For trend analysis we used the provided supplemental files that incorporate the newly calculated discharge weights using the 1998 NIS definition for the discharge population and available from the NIS-HCUP website (http://www.hcup-us. ahrq.gov/db/nation/nis/nistrends.jsp). Continuous data were presented as means and standard deviations or medians and 
Table 1 The ICD-9-CM classification of study cohort

\begin{tabular}{|c|c|c|}
\hline ICD9-CM code & Description & References \\
\hline $432.1,852.2,852.3$ & Subdural-hematoma & $(11,17,41,43)$ \\
\hline $518.5,518.82$ & Grand Mal Status & \\
\hline \multicolumn{3}{|l|}{$96.70,96.71,96.72$} \\
\hline $458.0,458.8,458.9,796.3,785.51,785.59$ & Cardiovascular dysfunction, hypotension, shock & -27 \\
\hline $286.2,286.6,286.9,287.3-5$ & $\begin{array}{l}\text { Hematological dysfunction, disseminated intravascular coagulation, purpura } \\
\text { fulminans, coagulopathy, thrombocytopenia }\end{array}$ & -27 \\
\hline $570,572.2,573.3$ & Hepatic dysfunction, acute hepatic failure, hepatic encephalopathy, hepatitis & -27 \\
\hline $584,580,585,39.95$ & $\begin{array}{l}\text { Renal dysfunction, acute renal failure, acute glomerulonephritis, renal shutdown, } \\
\text { hemodialysis }\end{array}$ & -27 \\
\hline \multirow[t]{2}{*}{$293,348.1,348.3,780.01,780.09,89.14$} & $\begin{array}{l}\text { Neurological dysfunction, transient organic psychosis, anoxic brain injury, } \\
\text { encephalopathy, coma. altered consciousness, electroencephalography }\end{array}$ & -27 \\
\hline & Comorbidities (Charlson et al. 1987) & $(13,27,30)$ \\
\hline 428.0-428.9 & Congestive heart failure & \\
\hline 401 & Hypertension & \\
\hline 249,250 & Diabetes mellitus & \\
\hline $491,492,496$ & COPD & \\
\hline 571 & Chronic liver failure & \\
\hline 585,586 & Chronic kidney disease & \\
\hline 196,199 & Cancer & \\
\hline
\end{tabular}

$\mathrm{SDH}, \mathrm{SE}$, co-morbidities, and acute organ dysfunctions used in this study.

inter-quartile ranges (IQRs), as appropriate. Categorical data was presented as proportions and 95\% confidence intervals (CIs). Odds ratios (Schulte et al. 2008) were calculated to determine independent predictors of hospital mortality. Given the dichotomous outcome, multiple logistic regression modeling was used. All factors of interest were included and parsimonious models were found by systematically removing the least significant factor and recalculating the model. The analysis was conducted using Structured Query Language (SQL) and the LME-4 package (ver. 0.99) in the $\mathrm{R}$ programming language for statistical computing (ver. 2.11), both available under the GNU Public License (http://cran.r-project.org/). Pearson chi-square test was used to determine statistical differences of corresponding demographic parameters between 1988 and 2011. P-values are two sided and statistical significance was judged when $p<0.05$. Our reporting of observational data conforms with Strengthening the Reporting of Observational Studies in Epidemiology STROBE guidelines (von Elm et al. 2007). Based on the de-identified nature of the database, the study was exempted from full IRB review.

\section{Results}

Over the 23-year period, we identified 1,583,255 admissions with a diagnosis of SDH. The prevalence of SE in this cohort was $0.5 \%$ (7,421 admissions). More than 50\% of admitted patients self-identified as white, with a male predominance of 59\%, (Table 2). The population adjusted incidence rate of admissions of SDH increased from 13 per 100,000 in 1988 to 38 per 100,000 in 2011, with a concomitant increase in the prevalence of SE diagnosis in this cohort, (Figure 1). More than half of admitted patients were treated in private institutions, with relatively equal geographic representation. Black patients had a statistically significantly higher prevalence of SE that their white counterparts (OR 1.71, $\mathrm{p}<0.001)$, (Table 3 ).

\section{Demographics}

There were no statistically significant difference in the age distribution between patients admitted with diagnosed SE compared to those who did not in this study group (SE; $65.53 \pm 17.1$, no SE; $69.64 \pm 18.07$ ). The gender distribution was also similar in both the SE and non-SE cohorts, (39.48\% and $41 \%$ female distribution respectively). The majority of patients admitted were identified as white $(50.67 \%$ vs $56.13 \%$ ), however among black patients there was a $50 \%$ greater prevalence of SE (Table 2).

\section{Morbidity and mortality}

Patients who presented with SE were more likely than not to have respiratory ( $36.4 \%$ vs $8.5 \%$ ), hematological $(10.8 \%$ vs $5.9 \%)$ or renal dysfunction $(20.1 \%$ vs $6.8 \%)$, (Table 2). Respiratory dysfunction was the strongest predictor of SE in this cohort (OR: 4.91, p <0.0001). The prevalence of SE among patients with SDH increased nominally, from $0.5 \%$ in 1988 to $0.7 \%$ in 2011, (Figure 2); 
Table 2 Demographic characteristics of the study cohort

\begin{tabular}{|c|c|c|c|c|}
\hline Variable & No status epilepticus & Percentage & With status epilepticus & Percentage \\
\hline Number of SDH patients & 1575834 & $99.50 \%$ & 7421 & 0.5 \\
\hline $1^{\circ} \mathrm{SDH} / 2^{\circ} \mathrm{SDH}$ & 1130031 / 445803 & $71.7 \% / 28.3 \%$ & $4337 / 3084$ & $58.4 \% / 41.6 \%$ \\
\hline Age [Mean + SD] & $69.64+18.07$ & & $65.53+17.1$ & \\
\hline Female/Male & 646092 / 929742 & $41 \% / 59 \%$ & $2930 / 4491$ & $39.5 \% / 60.5 \%$ \\
\hline \multicolumn{5}{|l|}{ Race $^{*}$} \\
\hline White & 884516 & $56.10 \%$ & 3760 & $50.70 \%$ \\
\hline Black & 127327 & $8.10 \%$ & 1147 & $15.50 \%$ \\
\hline Hispanic & 95653 & $6.10 \%$ & 581 & $7.80 \%$ \\
\hline Asian & 39711 & $2.50 \%$ & 141 & $1.90 \%$ \\
\hline Native American & 6303 & $0.40 \%$ & 59 & $0.80 \%$ \\
\hline Other & 30256 & $1.90 \%$ & 159 & $2.10 \%$ \\
\hline Not stated & 391910 & $24.90 \%$ & 1573 & $21.20 \%$ \\
\hline \multicolumn{5}{|l|}{ Hospital characteristics* } \\
\hline Rural & 111254 & $7 \%$ & 323 & $4.30 \%$ \\
\hline Urban private & 913669 & $58 \%$ & 4405 & $59.40 \%$ \\
\hline Urban academic & 550912 & $35 \%$ & 2693 & $36.30 \%$ \\
\hline Northeast & 307918 & $19.50 \%$ & 1479 & $19.90 \%$ \\
\hline Midwest & 352829 & $22.40 \%$ & 1523 & $20.50 \%$ \\
\hline South & 586368 & $37.20 \%$ & 2758 & $37.20 \%$ \\
\hline West & 328561 & $20.90 \%$ & 1660 & $22.40 \%$ \\
\hline Small to medium ( $<300$ beds) & 111096 & $7 \%$ & 470 & $6.40 \%$ \\
\hline Large (300-500 beds) & 330295 & $21 \%$ & 1633 & $22.00 \%$ \\
\hline Extra-large (>500 beds) & 1134285 & $72 \%$ & 5317 & $71.60 \%$ \\
\hline \multicolumn{5}{|l|}{ Major organ dysfunction } \\
\hline Respiratory & 133631 & $8.50 \%$ & 2701 & $36.40 \%$ \\
\hline Hematological & 92659 & $5.90 \%$ & 804 & $10.80 \%$ \\
\hline Hepatic & 11188 & $0.70 \%$ & 185 & $2.50 \%$ \\
\hline Neurological & 107472 & $6.80 \%$ & 1492 & $20.10 \%$ \\
\hline Metabolic & 26632 & $1.69 \%$ & 498 & $6.71 \%$ \\
\hline Renal & 137728 & $8.74 \%$ & 1322 & $17.82 \%$ \\
\hline Cardiovascular & 55154 & $3.50 \%$ & 555 & $7.48 \%$ \\
\hline \multicolumn{5}{|l|}{ Outcome } \\
\hline In hospital mortality & 210531 & $13.40 \%$ & 1937 & $26.10 \%$ \\
\hline
\end{tabular}

SDH: Sub-Dural Hematoma/*: Some Data is missing.

the diagnosis of $\mathrm{SE}$ in this sub-group was an independent predictor of in-hospital mortality (OR 2.17, p <0.0001), (Table 3). Over the course of study, there was a 7.6\% reduction in the hospital mortality rate among patients with SDH without recorded SE, from $17.9 \%$ in 1988 to $10.3 \%$ in 2011, (Figure 3). Throughout the course of study, there was a $4.1 \%$ decrease in hospital mortality rates among patients with both SDH and SE, from $27.8 \%$ in 1988 to $23.7 \%$ in 2011 (Figure 3). Independent predictors of in-hospital mortality among admissions with SDH are shown, (Table 4).

\section{Discussion}

In the cohort of patients admitted with a primary diagnosis of SDH, our assessment demonstrates an approximate three-fold increase in the incidence rate of admissions in the United States during the course of study. Similarly, while the prevalence of SE among admissions of SDH was low (0.5\%) there was a statistically significant increase in incidence rate of admissions of $\mathrm{SDH}$ over the 23 years. Most importantly, though the diagnosis of $\mathrm{SE}$ in this sub-set was an independent predictor of mortality, (OR 2.17, $\mathrm{p}<0.001)$ the in hospital 


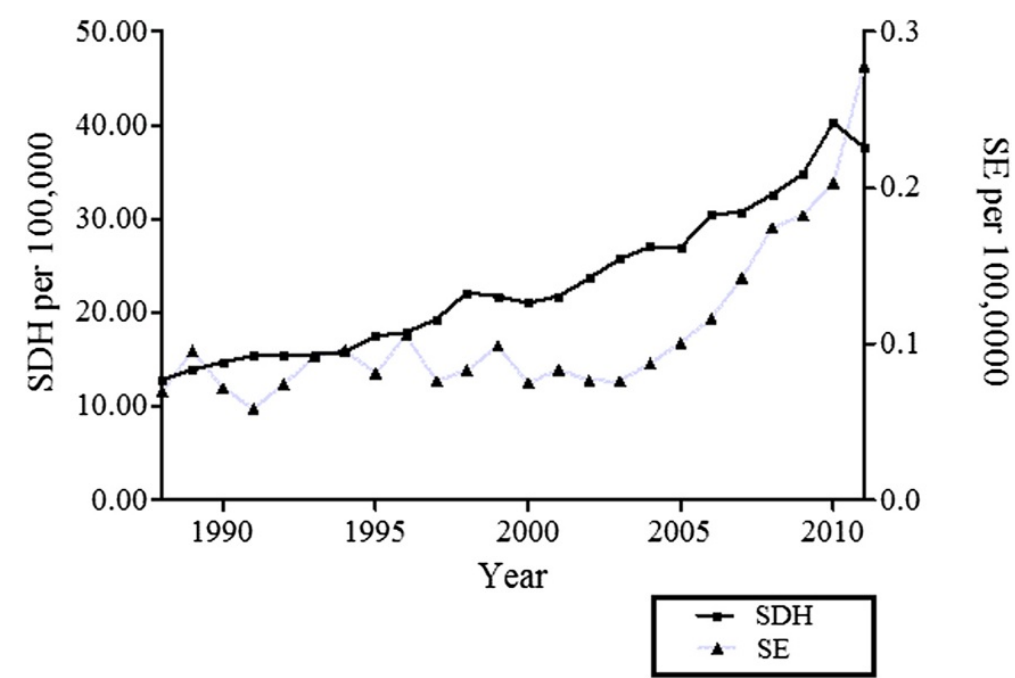

Figure 1 The population adjusted rate of subdural hematoma (SDH) hospitalizations from 1988 through 2011 in the USA.

mortality in the subset of patients with SE trended downwards over this time period but was not statistically significant. Comparatively, patients without SE demonstrated a statistically significant decrease in hospital mortality. Whether there was a significant mortality difference between these two sub-groups cannot be ascertained from current data.

Table 3 Predictors of status epilepticus among patients with subdural hematoma

\begin{tabular}{|c|c|c|c|c|}
\hline Variable & OR & $\begin{array}{l}95 \% \mathrm{Cl} \\
\text { (Lower) }\end{array}$ & $\begin{array}{l}95 \% \mathrm{Cl} \\
\text { (Upper) }\end{array}$ & P-Value \\
\hline Age (per year) & 0.99 & 0.99 & 0.99 & $<0.0001$ \\
\hline $1^{\circ} \mathrm{SDH}$ vs. $2^{\circ} \mathrm{SDH}$ & 0.73 & 0.65 & 0.83 & $<0.0001$ \\
\hline \multicolumn{5}{|l|}{ Race (vs white) } \\
\hline Black & 1.71 & 1.47 & 1.99 & $<0.0001$ \\
\hline Hispanic & 1.19 & 0.97 & 1.45 & 0.08 \\
\hline Asian & 0.79 & 0.54 & 1.13 & 0.1 \\
\hline Native American & 1.16 & 0.55 & 2.47 & 0.6 \\
\hline Other & 1.04 & 0.72 & 1.5 & 0.8 \\
\hline \multicolumn{5}{|l|}{$\begin{array}{l}\text { Hospital characteristics } \\
\text { (vs small to medium) }\end{array}$} \\
\hline Large & 1.05 & 0.82 & 1.34 & 0.6 \\
\hline Extra-large & 0.91 & 0.73 & 1.14 & 0.4 \\
\hline \multicolumn{5}{|l|}{ Major organ dysfunction } \\
\hline Hematologic dysfunction & 1.35 & 1.12 & 1.62 & 0.001 \\
\hline Metabolic dysfunction & 1.86 & 1.48 & 2.35 & $<0.0001$ \\
\hline Renal dysfunction & 1.47 & 1.25 & 1.72 & $<0.0001$ \\
\hline CNS dysfunction & 2.26 & 1.95 & 2.62 & $<0.0001$ \\
\hline Respiratory dysfunction & 4.91 & 4.31 & 5.58 & $<0.0001$ \\
\hline
\end{tabular}

Vs. Versus, SDH: Sub-Dural Hematoma.
In 2011, a retrospective analysis of SDH diagnoses as reported by the NIS demonstrated similar trends in admissions. Frontera et al showed a 39\% per capita increase in hospitalization for patients with SDH (Frontera et al. 2011). This increase in incidence has previously been attributed to an aging population in the USA (Frontera et al. 2011; Kudo et al. 1992; Santarius et al. 2009). However factors such as, improved diagnosis either due to physicians' evolving index of suspicion, and more available computed tomography (CT) scanners cannot be ruled out (Smith-Bindman et al. 2009; MPAC (U.S.) 2007; Amis et al. 2007). Similarly, we demonstrate a three-fold increase in the primary diagnosis of SDH patients who are hospitalized in the United States, (Figure 1) with no significant variance in the age distribution, (Figure 4).

The association between SDH and seizures and SE is repeatedly referenced in academic literature (Annegers et al. 1998; Rubin and Rappaport 1993; Cameron 1978; Drapkin 1991; Kotwica and Brzezinski 1991; Robinson 1984; Cole and Spatz 1961; Huang et al. 2011; Westmoreland 2001; Wiedemayer et al. 2002). On the contrary, large scale epidemiological studies of SE in this cohort is scarce. In our analyses, the prevlence of SE among SDH patients was low (0.5\%) with increasing prevalence over the course of study. It is possible that this may reflect an underdiagnoses in hospitalized patients either due to a low index of suspicion based on other clinical findings or insufficient use of EEG studies. Studies by two groups Rubin and Rappaport (1993) and Ohno et al (1993) demonstrated comparatively higher prevalence of seizures in their single institution cohorts; $5.6 \%$ and $2.3 \%$ respectively. More recently, a series of 100 patients with diagnosed SDH suggested an $11 \%$ rate in diagnosis of 


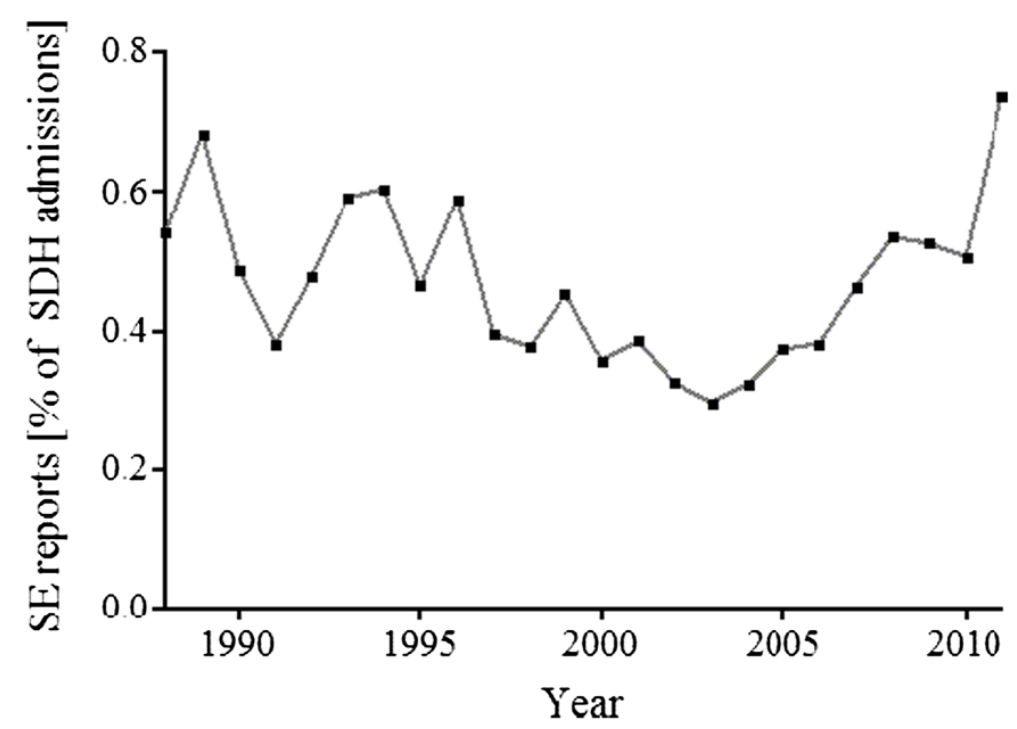

Figure 2 Prevalence of status epilepticus in admissions with subdural hematoma (SDH) from 1988 through 2011 in the USA.

inpatient seizure events, while there were no reported cases of SE (Huang et al. 2011). In a 2011 study, investigators retrospectively identified patients admitted with $\mathrm{SDH}$ who received surgical evacuation and electroencephalography (EEG) analysis. While $87 \%$ of this cohort had recorded epileptiform discharges, there were no reported cases of SE (Rudzinski et al. 2011). Quantitative improvement as diagnosed by EEG studies however correlated positively with improved long term functional outcome.
There are only a few case reports documenting SE in patients with SDH (Jones et al. 1989). The reason for this lower prevalence of SE among patients with SDH may be multi-factorial. Patients with trauma significant enough to cause SDH with post traumatic SE may have a higher mortality. We are unable to ascertain this relationship from NIS data. Additionally, for the identification of SE in the setting of SDH there needs to be a high clinical suspicion and in instances where patients are comatose secondary to significant injury, we recommend

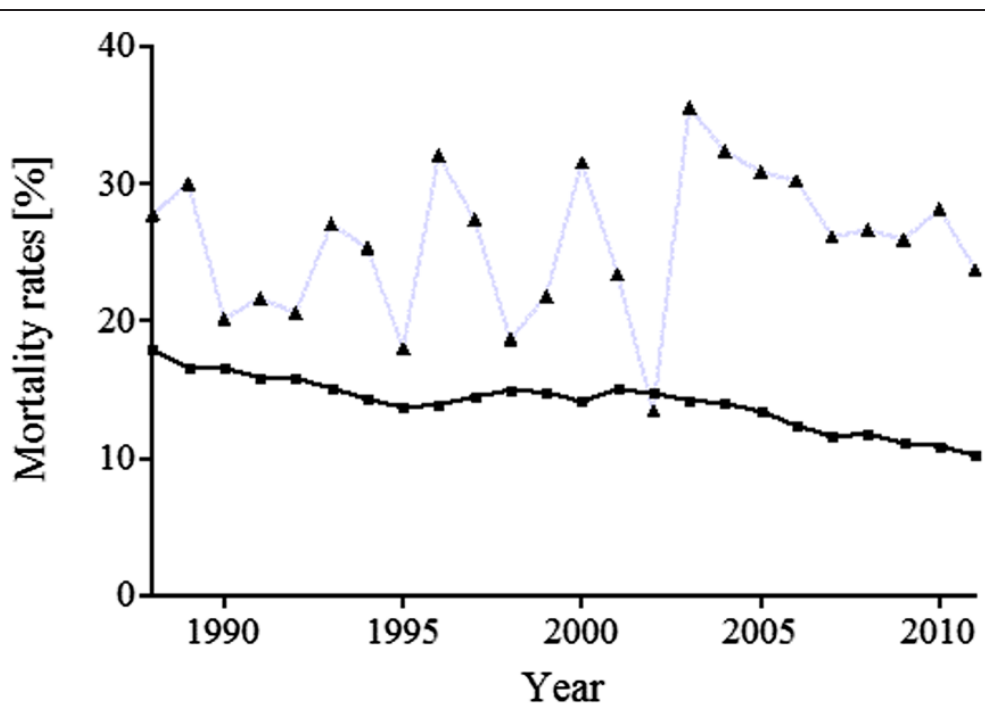

$\rightarrow$ SDH without SE

- SDH with SE

Figure 3 Mortality rates among admissions with SDH, with and without status epilepticus from 1988 through 2011 in the USA. 
Table 4 Predictors of in-hospital mortality among patients with subdural hematoma

\begin{tabular}{|c|c|c|c|c|}
\hline Variable & OR & $\begin{array}{l}95 \% \mathrm{Cl} \\
\text { (upper) }\end{array}$ & $\begin{array}{l}95 \% \mathrm{Cl} \\
\text { (Lower) }\end{array}$ & P-Value \\
\hline Status epilepticus & 0.71 & 0.55 & 0.91 & 0.007 \\
\hline Primary SDH & 1.06 & 1.03 & 2.95 & $<0.0001$ \\
\hline Status epilepticus in $1^{\circ} \mathrm{SDH}$ & 2.17 & 1.59 & 1.09 & $<0.0001$ \\
\hline Age (per year) & 1.01 & 1.01 & 1.1 & $<0.0001$ \\
\hline Female (vs. male) & 1.07 & 1.05 & 1.01 & $<0.0001$ \\
\hline \multicolumn{5}{|l|}{ Race } \\
\hline Black (vs. white) & 0.83 & 0.8 & 1.01 & $<0.0001$ \\
\hline Hispanic(vs. white) & 0.83 & 0.79 & 1.07 & $<0.0001$ \\
\hline Asian(vs. white) & 0.88 & 0.82 & 0.95 & 0.001 \\
\hline Native American (vs. white) & 0.88 & 0.73 & 0.87 & 0.2 \\
\hline Other (vs. white) & 0.93 & 0.86 & 0.87 & 0.08 \\
\hline \multicolumn{5}{|l|}{ Hospital Characteristics } \\
\hline Midwest (vs. Northeast) & 0.91 & 0.87 & 0.88 & $<0.0001$ \\
\hline South (vs. Northeast) & 0.94 & 0.91 & 0.97 & $<0.0001$ \\
\hline West (vs. Northeast) & 0.84 & 0.81 & 0.95 & $<0.0001$ \\
\hline Large (vs. small to medium) & 1.18 & 1.11 & 1.16 & $<0.0001$ \\
\hline Extra-large (vs. small to medium) & 1.18 & 1.13 & 1.3 & $<0.0001$ \\
\hline Rural (vs. academic) & 1.23 & 1.16 & 1.24 & $<0.0001$ \\
\hline Urban private (vs. academic) & 1.13 & 1.1 & 1.24 & $<0.0001$ \\
\hline \multicolumn{5}{|l|}{ Major Organ Dysfunction } \\
\hline Cardiovascular dysfunction & 2.35 & 2.23 & 2.47 & $<0.0001$ \\
\hline Hematologic dysfunction & 2.13 & 2.04 & 2.23 & $<0.0001$ \\
\hline Metabolic dysfunction & 1.86 & 1.73 & 2.01 & $<0.0001$ \\
\hline Renal dysfunction & 1.68 & 1.62 & 1.75 & $<0.0001$ \\
\hline Hepatic dysfunction & 1.64 & 1.46 & 5.18 & $<0.0001$ \\
\hline CNS dysfunction & 2.03 & 1.95 & 2.11 & $<0.0001$ \\
\hline Respiratory dysfunction & 5.01 & 4.85 & 1.85 & $<0.0001$ \\
\hline
\end{tabular}

SDH: Sub-Dural Hematoma, vs.: Versus.

the use of continuous EEG recordings for diagnosis. Though the most recent European Society of Intensive Care Medicine (ESICM) guidelines strongly suggest incorporation of EEG assessments in this patient population (Claassen et al. 2013), low subscribership will result in under diagnosis. The underlying theory for epileptogenicity is hinged on the principle that acute ischemia in addition to exposure to cytotoxic mediators is the basis for neuronal injury and subsequent excitability (Shaver et al. 1996; Sahuquillo-Barris et al. 1988; Becker 1986). It follows that patients with an intact pial-arachnoid layer are less likely to develop PTE and SE (Tomkins et al. 2008). Whether patients who develop SE after SDH present with more leptomeningeal disruption than those who do not warrants further study.
Self-identified black patients were more likely to have a diagnosis of SE in the setting of SDH than other ethnic groups, Table 2. Asians on the other hand had a lower risk of SE in our study group. In previous studies, it has also been observed that the prevalence of epilepsy and $\mathrm{SE}$ is generally higher in African-Americans than other ethnic groups in the United States (Haerer et al. 1986; Logroscino et al. 2005; DeLorenzo et al. 1995). The reasons behind this finding is not well understood and warrants further studies.

Major organ dysfunction was correlated to the diagnosis of SE. Patients with a diagnosis of SDH were most likely to have SE if they presented with respiratory, renal or neurological dysfunctions, (Table 2). Whether or not this observation is related to acute co-existing systemic injury is currently unknown. It is also possible that these factors directly and indirectly affect the cost of disposition in addition to discharge morbidity in these patients. In 2011, Frontera and colleagues demonstrated increased hospital costs in patients presenting with multi-system injuries (Frontera et al. 2011). Consequently the diagnosis and treatment of any systemic derangement in patients with SDH may impact functional outcome in these patients.

This is the first assessment of the morbidity and mortality attendant to patients co-diagnosed with SE and $\mathrm{SDH}$ from data report in the NIS database. We demonstrate that SE in patients with a diagnosis of SDH is associated with an increased risk of mortality (OR 2.17, $\mathrm{p}<0.001$ ); a finding consistent with smaller studies (Sabo et al. 1995; Sung and Chu 1989; Claassen et al. 2003). We are unable to assess the impact or surgical intervention in the subset of patients with surgically correctable lesions, or the severity of TBI in the subset who presented after acute brain injury due to limitations of the dataset. However, in a retrospective assessment of patients undergoing SDH clot evacuation, Rabinstein and colleagues demonstrated a high incidence of epileptiform activity characterized by EEG recordings, (25\%); SE was not reported in any case. Interestingly however, in their study, the presence of epileptiform discharges on EEG recordings were independently associated with poor postoperative outcome, but not long term functional recovery (Rabinstein et al. 2010). It is important to note that in patients with epileptiform activity anti-epileptic treatment was initiated (Rabinstein et al. 2010). Future studies assessing the impact of surgical intervention and the severity of trauma on SE should be undertaken.

From a public health standpoint, in critically ill patients with severe TBI and SDH the diagnosis and treatment of SE via continuous EEG monitoring has a positive mortality benefit (Bleck 2012), and should be a fixture in the management of this patient population. The treatment of electrocorticographic abnormalities with prophylactic AEDs may also have clinical benefit in some subsets of this 


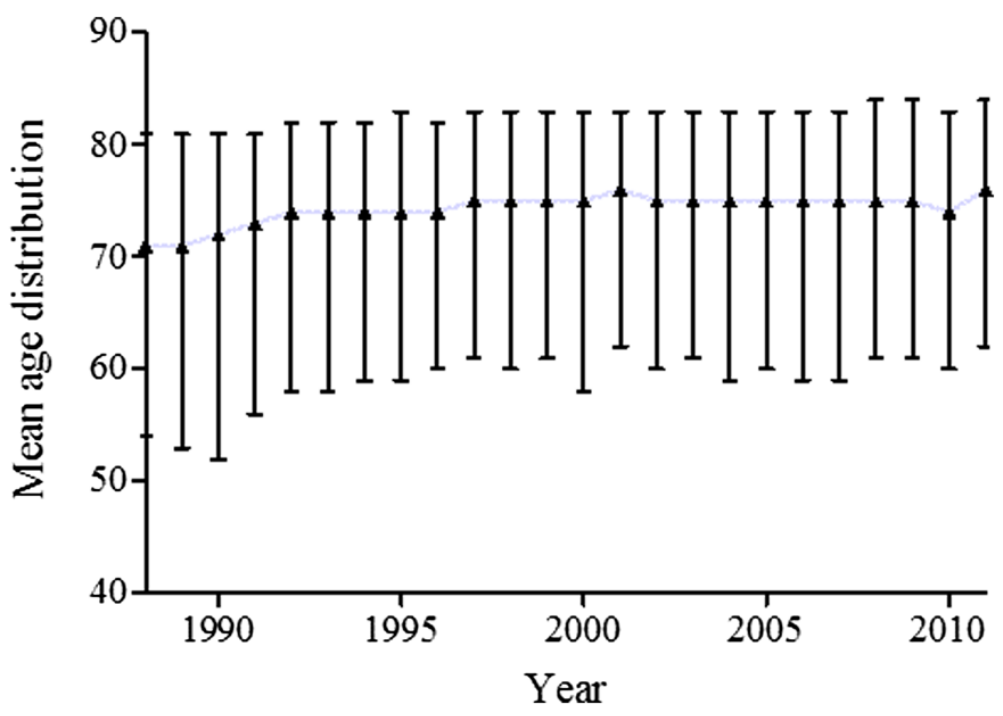

Figure 4 Variance in the age distribution in subdural hematoma (SDH) hospitalizations from 1988 through 2011 in the USA.

patient population. In a recently published retrospective study, researchers demonstrated clinical efficacy in using either leviteracetam (LVT) or phenytoin for seizure prophylaxis in patients with acute or subacute SDH. One salient differences between the two cohorts was increased side effects historically attributable to phenytoin usage, when compared to the cohort treated with LVT (Radic et al. 2014). Contrastingly, Rappaport in 1993 reported the absence of evidence for AED usage in patients presenting with non-alcoholic patients with chronic SDH (Rubin and Rappaport 1993).

\section{Limitations of the study}

This study has limitations which implicitly affects its interpretation. ICD-9 codes have questionable accuracy, since they may change over time (Frontera et al. 2011; Claassen et al. 2007; Urtecho et al. 2013). Secondly, the ICD-9-CM does not allow for differentiation between acute, chronic and sub-acute SDH which have differing risk factors, morbidity and mortality profiles. This study is an observational study thus not as robust as prospective analyses; neither can we assume etiological or causal relationships between variables and outcomes. Additionally, the NIS is an inpatient database representing an approximate stratified $20 \%$ sample of all non-federal, short-term, general, and specialty hospitals serving adults in the United States. This may not be an accurate representation of national trends in instances of under-reporting or under-diagnosis. As highlighted in similar studies, the data recorded in the NIS database includes both clinical and electroencephalographic diagnoses of SE without clear differentiation. The absence of standardization in the diagnosis of SE, introduces observer biases that cannot be corrected for in this study (Urtecho et al. 2013). Surgical interventions such as burr-hole craniotomies or trephination have been shown to affect morbidity after SDH (Pahatouridis et al. 2013; Krupp and Jans 1995). These variables were not assessed in our study due to the limitations of the dataset but may impact overall mortality/morbidity rates. Long term outcomes secondary to the diagnosis of SDH or SE were not assessed in this study; therefore it can be argued that long outcomes after discharge may differ from our reported data. The NHDS does not allow evaluation of various other variables such as other comorbidities, rates of DNR orders, timing of therapeutics and time of onset. These variables may have a confounding impact on our data. There may be patients with prior diagnoses of seizure disorders represented in this dataset. Since the NIS describes comorbidities upon patient discharge it is impossible to differentiate new diagnoses of seizure disorders from pre-admission diagnoses.

\section{Conclusions}

Our study demonstrates an approximate three-fold increase in the incidence of SDH during the study period in the United States of America; however from our data the incidence of SE among patients with SDH was relatively low, (0.5\%). The risk of SE among patients with $\mathrm{SDH}$ increased with age, and higher in African-Americans and in those with respiratory, hematological, or renal dysfunction. Despite a decline in the overall SDH related mortality, SE increased the risk of in-hospital death in patients with primary SDH during the study period. Increased utilization of EEG studies may positively impact in-hospital mortality through improved diagnosis in this patient cohort. 


\section{Abbreviations}

SE: Status epilepticus; SDH: Subdural hematoma; CT: Computed tomarized; NIS: Nationwide inpatient sample; PTE: Post traumatic epilepsy.

\section{Competing interests}

Authors declare that they have no competing interests.

\section{Authors' contributions}

AS: Writing the manuscript, search the literature, editing. AA: Writing the article. KC: Writing the article. MM: Statistical analysis. ME: Writing the article. RB: Search the literature, writing the article. MM: Writing the article. MY: Search the literature. FR: Main topic idea, principal investigator. All authors read and approved the final manuscript.

\section{Authors' information}

Ali Seifi, M.D., FACP: Assistant Professor of Neurosurgery, Neuro Critical Care. University of Texas Health Science Center at San Antonio, USA. Ali Akbar Asadi-Pooya, M.D: Associate Professor of Epileptology. Neurosciences Research Center, Shiraz University of Medical Sciences, Shiraz, Iran. Kevin Carr, M.D: Neuorsurgery Resident. University of Texas Health Science Center at San Antonio, USA. Mitchell Maltenfort, Ph.D: Statistician. The Rothman Institute, Philadelphia, USA. Mehrdad Emami, M.D: General Physician. Shiraz University of Medical Sciences, Shiraz, Iran Rodney Bell, M.D: Professor of Neurology and Neurosurgery. Thomas Jefferson University, Philadelphia, USA. Michael Moussouttas, M.D: Clinical Neurointensivist. Capital Institute of Neurosciences, Trenton, USA. Moussa Yazbeck, M.D., FACP: Clinical Neurointensivist, Department of Neurosurgery, John Muir Medical Center, Walnut Creek, USA Fred Rincon, M.D., FACP, FCCP, FCCM: Assistant Professor of Neurosurgery and Neurology. Thomas Jefferson University, Philadelphia, USA.

\section{Author details}

${ }^{1}$ Department of Neurosurgery, Division of Neurocritical Care, University of Texas Health Science Center at San Antonio, Mail Code 7843, 7703 Floyd Curl Drive, Medical School Building 102F, San Antonio, TX 78229-3900, USA. ${ }^{2}$ Neurosciences Research Center, Shiraz University of Medical Sciences, Shiraz, Iran. ${ }^{3}$ Jefferson Comprehensive Epilepsy Center, Department of Neurology, Thomas Jefferson University, Philadelphia, USA. ${ }^{4}$ The Rothman Institute, Philadelphia, PA, USA. ${ }^{5}$ Department of Neurology and Neurosurgery, Thomas Jefferson University, Philadelphia, USA. ${ }^{6}$ Division of Neuro Critical Care, Capital Institute for Neurosciences, Trenton, USA. 'Department of Neurosurgery, John Muir Medical Center, Walnut Creek, USA.

Received: 10 April 2014 Accepted: 24 June 2014

Published: 1 July 2014

\section{References}

Agrawal A, Timothy J, Pandit L, Manju M (2006) Post-traumatic epilepsy: an overview. Clin Neurol Neurosurg 108:433-439

Amis ESJ, Butler P, Applegate K, Birnbaum SB, Brateman LF, Hevezi JM, Mettler FA, Morin RL, Pentecost MJ, Smith GG, Strauss KJ, Zeman RK, American College of Radiology (2007) American college of radiology white paper on radiation dose in medicine. J Am Coll Radiol 4:272-284

Annegers JF, Hauser WA, Coan SP, Rocca WA (1998) A population-based study of seizures after traumatic brain injuries. N Engl J Med 338:20-24

Balser D, Rodgers SD, Johnson B, Shi C, Tabak E, Samadani U (2013) Evolving management of symptomatic chronic subdural hematoma: experience of a single institution and review of the literature. Neurol Res 35:233-242

Becker DP (1986) Acute subdural hematomas. Advances in Neurotraumatology $1: 51-100$

Bleck T (2012) Status epilepticus and the use of continuous EEG monitoring in the intensive care unit. Continuum (Minneap Minn) 18:560-578

Brodersen P, Gjerris F (1975) Regional cerebral blood flow in patients with chronic subdural hematomas. Acta Neurol Scand 51:233-239

Byung-Soo K, Jung-Kil L, Bo-Ra S, Sung-Jun M, Jae-Hyoo K, Soo-Han K (2008) Clinical analysis of risk factors related to recurrent chronic subdural hematoma. J Korean Neurosurg Soc 43:11-15

Cameron M (1978) Chronic subdural haematoma: a review of 114 cases. J Neurol Neurosurg Psychiatry 41:834-839

Charlson ME, Pompei P, Ales KL, MacKenzie CR (1987) A new method of classifying prognostic comorbidity in longitudinal studies: development and validation. J Chronic Dis 40:373-383
Claassen J, Peery S, Kreiter K, Hirsch LJ, Du EY, Connolly ES, Mayer SA (2003) Predictors and clinical impact of epilepsy after subarachnoid hemorrhage. Neurology 60:208-214

Claassen J, Hirsch LJ, Frontera JA, Fernandez A, Schmidt M, Kapinos G, Wittman J, Connolly ES, Emerson RG, Mayer SA (2006) Prognostic significance of continuous EEG monitoring in patients with poor-grade subarachnoid hemorrhage. Neurocrit Care 4:103-112

Claassen J, Bateman BT, Willey JZ, Inati S, Hirsch LJ, Mayer SA, Sacco RL, Schumacher HC (2007) Generalized convulsive status epilepticus after nontraumatic subarachnoid hemorrhage: the nationwide inpatient sample. Neurosurgery 61:60-64, discussion 4-5

Claassen J, Taccone FS, Horn P, Holtkamp M, Stocchetti N, Oddo M (2013) Recommendations on the use of EEG monitoring in critically ill patients: consensus statement from the neurointensive care section of the ESICM. Intensive Care Med 39:1337-1351

Cole M, Spatz E (1961) Seizures in chronic subdural hematoma. N Engl J Med 265:628-631

DeLorenzo R, Pellock J, Towne A, Boggs J (1995) Epidemiology of status epilepticus. J Clin Neurophysiol 12:316-325

Dennis LJ, Claassen J, Hirsch LJ, Emerson RG, Connolly ES, Mayer SA (2002) Nonconvulsive status epilepticus after subarachnoid hemorrhage. Neurosurgery 51:1136-1143, discussion 44

Drapkin A (1991) Chronic subdural hematoma: pathophysiological basis for treatment. Br J Neurosurg 5:467-473

Englander J, Bushnik T, Duong TT, Cifu DX, Zafonte R, Wright J, Hughes R, Bergman W (2003) Analyzing risk factors for late posttraumatic seizures: a prospective, multicenter investigation. Arch Phys Med Rehabil 84:365-373

Frontera J, Egorova N, Moskowitz A (2011) National trend in prevalence, cost, and discharge disposition after subdural hematoma from 1998-2007. Crit Care Med 39:1619-1625

Gupta YK, Gupta M (2006) Post traumatic epilepsy: a review of scientific evidence. Indian J Physiol Pharmacol 50:7-16

Haerer A, Anderson D, Schoenberg B (1986) Prevalence and clinical features of epilepsy in a biracial United States population. Epilepsia 27:66-75

Halpern NA, Pastores SM, Thaler HT, Greenstein RJ (2006) Changes in critical care beds and occupancy in the United States 1985-2000: differences attributable to hospital size. Crit Care Med 34:2105-2112

Huang YH, Yang TM, Lin YJ, Tsai NW, Lin WC, Wang HC, Chang WN, Lu CH (2011) Risk factors and outcome of seizures after chronic subdural hematoma. Neurocrit Care 14:253-259

Hung C, Chen JW (2012) Treatment of post-traumatic epilepsy. Curr Treat Options Neurol 14:293-306

Jones S, Bamford J, Heath J, Bradey N, Heatley R (1989) Multiple forms of epileptic attack secondary to a small chronic subdural haematoma. BMJ 299:439-441

Kotwica Z, Brzezinski J (1991) Chronic subdural haematoma treated by burr holes and closed system drainage: personal experience in 131 patients. $\mathrm{Br} \mathrm{J}$ Neurosurg 5:461-465

Krupp WF, Jans PJ (1995) Treatment of chronic subdural haematoma with burr-hole craniostomy and closed drainage. Br J Neurosurg 9:619-627

Kudo H, Kuwamura K, Izawa I, Sawa H, Tamaki N (1992) Chronic subdural hematoma in elderly people: present status on Awaji island and epidemiological prospect. Neurol Med Chir 32:207-209

Little AS, Kerrigan JF, McDougall CG, Zabramski JM, Albuquerque FC, Nakaji P, Spetzler RF (2007) Nonconvulsive status epilepticus in patients suffering spontaneous subarachnoid hemorrhage. J Neurosurg 106:805-811

Logroscino G, Hesdorffer D, Cascino G, Hauser WA, Coeytaux A, Galobardes B, Morabia A, Jallon P (2005) Mortality after a first episode of status epilepticus in the United States and Europe. Epilepsia 46:46-48

MPAC (U.S.) (2007) Report to the Congress: Promoting Greater Efficiency in Medicare. MedPAC, Washington, DC

Ohno K, Maehara T, Ichimura K, Suzuki R, Hirakawa K, Monma S (1993) Low incidence of seizures in patients with chronic subdural haematoma. J Neurol Neurosurg Psychiatry 56:1231-1233

Pahatouridis D, Alexiou GA, Fotakopoulos G, Mihos E, Zigouris A, Drosos D, Voulgaris S (2013) Chronic subdural haematomas: a comparative study of an enlarged single burr hole versus double burr hole drainage. Neurosurg Rev 36:151-154, discussion 4-5

Payan H, Toga M, Berard-Badier M (1970) The pathology of post-traumatic epilepsies. Epilepsia 11:81-94

Quan H, Sundararajan V, Halfon P, Fong A, Burnand B, Luthi JC, Saunders LD, Beck CA, Feasby TE, Ghali WA (2005) Coding algorithms for defining comorbidities in ICD-9-CM and ICD-10 administrative data. Med Care 43:1130-1139 
Rabinstein AA, Chung SY, Rudzinski LA, Lanzino G (2010) Seizures after evacuation of subdural hematomas: incidence, risk factors, and functional impact. J Neurosurg 112:455-460

Radic JA, Chou SH, Du R, Lee JW (2014) Levetiracetam versus phenytoin: a comparison of efficacy of seizure prophylaxis and adverse event risk following acute or subacute subdural hematoma diagnosis. Neurocrit Care, doi:10.1007/s12028-013-9951-x

Rincon F, Ghosh S, Dey S, Maltenfort M, Vibbert M, Urtecho J, McBride W, Moussouttas M, Bell R, Ratliff JK, Jallo J (2012) Impact of acute lung injury and acute respiratory distress syndrome after traumatic brain injury in the United States. Neurosurgery 71:795-803

Robinson R (1984) Chronic subdural hematoma: surgical management in 133 patients. J Neurosurg 61:263-268

Rubin G, Rappaport Z (1993) Epilepsy in chronic subdural haematoma. Acta Neurochir 123:39-42

Rubin JJ, Willmore $\sqcup$ (1980) Prevention of iron-induced epileptiform discharges in rats by treatment with antiperoxidants. Exp Neurol 67:472-480

Rudzinski L, Rabinstein A, Chung S, Wong-Kisiel LC, Burrus TM, Lanzino G, Westmoreland BF (2011) Electroencephalographic findings in acute subdural hematoma. J Clin Neurophysiol 28:633-641

Sabo RA, Hanigan WC, Aldag JC (1995) Chronic subdural hematomas and seizures: the role of prophylactic anticonvulsive medication. Surg Neurol 43:579-582

Sahuquillo-Barris J, Lamarca-Ciuro J, Vilalta-Castan J, Rubio-Garcia E, RodriguezPazos M (1988) Acute subdural hematoma and diffuse axonal injury after severe head trauma. J Neurosurg 68:894-900

Santarius T, Kirkpatrick P, Ganesan D, Chia HL, Jalloh I, Smielewski P, Richards HK, Marcus H, Parker RA, Price SJ, Kirollos RW, Pickard JD, Hutchinson PJ (2009) Use of drains versus no drains after burr-hole evacuation of chronic subdural haematoma: a randomised controlled trial. Lancet 374:1067-1073

Schulte S, Donas KP, Pitoulias GA, Horsch S (2008) Endovascular treatment of iatrogenic and traumatic carotid artery dissection. Cardiovasc Intervent Radiol 31:870-874

Shaver EG, Duhaime A-C, Curtis M, Gennarelli LM, Barrett R (1996) Experimental acute subdural hematoma in infant piglets. Pediatr Neurosurg 25:123-129

Smith-Bindman R, Lipson J, Marcus R, Kim KP, Mahesh M, Gould R, Berrington de González A, Miglioretti DL (2009) Radiation dose associated with common computed tomography examinations and the associated lifetime attributable risk of cancer. Arch Intern Med 169:2078-2086

Steg RE (1993) Post-traumatic epilepsy. Nebr Med J 78:27-29

Storti SF, Formaggio E, Franchini E, Bongiovanni LG, Cerini R, Fiaschi A, Michel CM, Manganotti P (2012) A multimodal imaging approach to the evaluation of post-traumatic epilepsy. Magma 25:345-360

Sung C, Chu N (1989) Status epilepticus in the elderly: Etiology, seizure type and outcome. Acta Neurol Scand 80:51-56

Tomkins O, Shelef I, Kaizerman I, Eliushin A, Afawi Z, Misk A, Gidon M, Cohen A, Zumsteg D, Friedman A (2008) Blood-brain barrier disruption in posttraumatic epilepsy. J Neurol Neurosurg Psychiatry 79:774-777

Urtecho J, Snapp M, Sperling M, Maltenfort M, Vibbert M, Athar MK, McBride W, Moussouttas M, Bell R, Jallo J, Rincon F (2013) Hospital mortality in primary admissions of septic patients with status epilepticus in the United States*. Crit Care Med 41:1853-1862

von Elm E, Altman DG, Egger M, Pocock SJ, Gotzsche PC, Vandenbroucke JP (2007) The Strengthening the Reporting of Observational Studies in Epidemiology (STROBE) statement: guidelines for reporting observational studies. Lancet 370:1453-1457

Westmoreland B (2001) Periodic lateralized epileptiform discharges after evacuation of subdural hematomas. J Clin Neurophysiol 18:20-24

Wiedemayer $\mathrm{H}$, Triesch K, Schafer $\mathrm{H}$, Stolke D (2002) Early seizures following non-penetrating traumatic brain injury in adults: risk factors and clinical significance. Brain Inj 16:323-330

Willmore L, Sypert GW, Munson JB (1978) Recurrent seizures induced by cortical iron injection: a model of posttraumatic epilepsy. Ann Neurol 4:329-336

Yeh CC, Chen TL, Hu CJ, Chiu WT, Liao CC (2013) Risk of epilepsy after traumatic brain injury: a retrospective population-based cohort study. J Neurol Neurosurg Psychiatry 84:441-445

doi:10.1186/2193-1801-3-332

Cite this article as: Seifi et al:: The epidemiology, risk factors, and impact on hospital mortality of status epilepticus after subdural hematoma in the United States. SpringerPlus 2014 3:332

\section{Submit your manuscript to a SpringerOpen ${ }^{\circ}$ journal and benefit from:}

- Convenient online submission

- Rigorous peer review

- Immediate publication on acceptance

- Open access: articles freely available online

- High visibility within the field

- Retaining the copyright to your article

Submit your next manuscript at $>$ springeropen.com 